\title{
Penetrance and expressivity in the molecular age
}

Joël Zlotogora, $M D, P h D$

\begin{abstract}
Penetrance and expressivity have been defined through clinical experience. Although penetrance is often seen as the end of the spectrum of expressivity, penetrance and expressivity are considered as distinct phenomena. A review of the known mechanisms underlying either penetrance or expressivity reveals that in most of the cases the same explanation is true for both phenomena. Some of the known mechanisms include modifier genes, the influence of the allele in trans, sex, and environmental factors. Although rapid progress has been made in understanding of the basis of incomplete penetrance and the differences of expressivity, they still remain unknown for most of the genetic disorders. In recent years, it has become evident that there is much in common between the classical Mendelian traits in which the inheritance has been seen as "simple" and most of the common diseases in which the inheritance is "complex." In both cases genetic and/or environmental factors are acting in a complex way. Genet Med 2003:5(5):347-352.
\end{abstract}

Key Words: penetrance, expressivity, monogenic, Mendelian inheritance

Penetrance is used to describe whether or not there is a clinical expression of the genotype in the individual. Expressivity is the term that describes the differences observed in the clinical phenotype between two individuals with the same genotype. With the advances in molecular genetics over the last few years, some of the underlying mechanisms have been unraveled.

\section{PENETRANCE}

"A genotype which may or may not produce a clinical trait is said to be incompletely penetrant." The penetrance of a genotype is defined by the probability that a person carrying it will present clinical manifestations. Only after a complete examination finds no clinical signs of the syndrome in an obligate carrier, may it be concluded that the mutation is nonpenetrant in that individual. Penetrance has been until recently a term used almost exclusively for dominant disorders because analysis of the pedigrees alone allowed one to diagnose healthy obligatory carriers. Incomplete penetrance was difficult to detect in recessive disorders because it is enough to be a carrier to transmit the disease. In recessive disorders, incomplete penetrance was suspected only in large pedigrees when the segregation ratio was significantly lower than the expected 0.25 after correction of the ascertainment bias. Recently, the existence of healthy homozygotes of recessive disorders has been demonstrated by molecular analysis showing that incomplete penetrance is also relevant in recessive disorders (Table 1).

From the Department of Community Genetics, Public health services, Ministry of Health, Israel.

Joël Zlotogora, MD, PhD, Department of Community Genetics, Public Health Services, Ministry of Health, Israel.

Received: March 17, 2003

Accepted: May 21, 2003.

DOI: 10.1097/01.GIM.0000086478.87623.69

\section{"Pseudo-incomplete penetrance"}

The definition of nonpenetrance depends upon the accuracy of the clinical phenotype analysis. In some cases, the observation of nonpenetrance is inaccurate because the clinical examination is incomplete, or the symptoms have not yet appeared at the time of the examination. Germ line mosaicism is a particular example of pseudo-incomplete penetrance that is seen only in the first generation when the parents of several children affected with a dominant disease are healthy. The absence of symptoms in the parents is due to the presence of a mutation in the germ cells with or without a mosaicism in the somatic cells. $^{2}$

\section{Facioscapulohumeral muscular dystrophy}

Facioscapulohumeral muscular dystrophy is an autosomal dominant disease with progressive muscular weakness with a characteristic pattern of involvement. ${ }^{3}$ More than $90 \%$ of the patients demonstrate clinical symptoms by the age of 20 years with higher penetrance in males. The disease is often caused by rearrangements in the gene FSHD1A. Several families have been reported in which both healthy parents with no familial history had more than one affected child. Molecular studies demonstrated that one of the healthy parents carried the mutation as a mosaicism in the germ line. ${ }^{2}$

\section{Premutation}

The incomplete penetrance in this case is due to the existence of an intermediate state of the mutation, with no clinical expression. The mutation may change from one generation to another and therefore a normal individual with a premutation may have several affected children in whom the mutation is fully penetrant. The hallmark of this type of inheritance is that incomplete penetrance is present only in the early generations 
Table 1

Examples of mechanisms explaining incomplete penetrance

"Pseudo-incomplete penetrance"

Premutation

Sex limitation

Influence of the allele in trans

Influence of a modifier gene

Digenic inheritance

Imprinting

Influence of environmental factors

whereas subsequent generations of affected individuals will show complete penetrance in their children.

\section{Fragile $X$ syndrome}

The fragile $\mathrm{X}$ syndrome is the most common form of $\mathrm{X}$ linked mental retardation. The clinical expression of the disease is variable with mental retardation being the most common feature. In general, mental retardation is more severe in the affected males than in the affected females. The observation that many obligatory carriers in families with the fragile X syndrome are healthy was made before the knowledge of its molecular basis. Sherman et al. ${ }^{4}$ reported the paradoxical observation that among the children of normal carriers, the risk for mental retardation depends upon the sex of the carrier parent. Whereas the children of female carriers are often mentally retarded, the daughters of normal transmitting males are normal even though they are obligatory carriers of the mutation. The incomplete penetrance has been explained after the cloning of the FMR1 gene by the existence of premutation carriers who are clinically normal. ${ }^{5}$ The premutation expands to a full mutation almost exclusively through a female carrier.

\section{Sex limitation}

For some mutations, the clinical symptoms are present only in one sex. Sex limitation is often seen in disorders in which the expression of the disease is limited to organs found in only one of the sexes, such testis or ovaries.

\section{Steroid $5 \alpha$ reductase deficiency}

This is an autosomal recessive disorder in which the homozygous females are normal and the homozygous males are either born with ambiguous genitalia or almost normal external female genitalia. ${ }^{6}$ During childhood the affected boys undergo various degrees of virilization. The mutated enzyme $5 \alpha$ reductase catalyzes the conversion of testosterone to dehydrotestosterone in androgen sensitive tissues. Therefore, although affected males will present with abnormal external genitalia, the affected females will be normal.

\section{Influence of the allele in trans}

In a dominant disorder, the clinical phenotype is present in the heterozygote. Either a polymorphism or a mutation of the allele in trans may lead to the incomplete penetrance of the dominant mutation.

\section{Retinitis pigmentosa}

Progressive visual loss, night blindness, and pigmentary deposition in the retina characterize retinitis pigmentosa. Retinitis pigmentosa is genetically heterogeneous and has been associated with many genetic loci. ${ }^{7}$ In autosomal dominant retinitis pigmentosa due to mutations at the locus RP 11 (19q13.4, gene RPP31), the penetrance is incomplete. A segregation study in families with a RP11 mutation demonstrated that the segregation of the allele from the noncarrier parent is not at random. ${ }^{8}$ This observation was highly significant and strongly suggests an influence of the wild-type allele on the expression of the mutated allele.

\section{Autosomal-dominant glaucoma}

Glaucoma is a leading cause of blindness and is usually classified according to the anatomy and/or the age of onset. As a rule, the earlier the onset, the more severe the visual impairment. In a large pedigree with autosomal-dominant glaucoma caused by a mutation in the MYOC (TIGR) gene, several homozygotes for the mutation were diagnosed by molecular studies among the children of two affected individuals. ${ }^{9}$ None of these children had any clinical symptoms of glaucoma. This was significantly different from the expected as observed in the other adult carriers of the mutation in the family when adjusted for age. This finding is probably secondary to a dominant-negative effect of the mutation.

\section{Influence of a modifier gene}

The presence of a mutation/polymorphism at a locus different from the one causing the disease may alter the effect of the mutation and prevent its clinical manifestations.

\section{Autosomal-recessive deafness DFNB26}

Many different gene loci have been reported to be responsible for nonsyndromic autosomal recessive hearing loss. In a large pedigree with autosomal-recessive deafness, linkage to the chromosomal region $4 \mathrm{q} 31$ was demonstrated. ${ }^{10}$ However, whereas all the affected individuals were homozygous for the same haplotype, homozygosity was also found in many unaffected siblings. By linkage analysis, the authors were able to demonstrate the existence of a dominant modifier (DFNM1) on chromosome 1q24. The presence of this modifier in an individual homozygous for the DFNB26 mutation leads to nonpenetrance and normal hearing.

\section{Digenic inheritance}

The term digenic inheritance was proposed to describe a situation in which the clinical expression depends upon the presence of two mutations in two different genes.

\section{Retinitis pigmentosa}

Mutations in the gene peripherin RDS are responsible for either autosomal recessive or dominant retinitis pigmentosa. ${ }^{11}$ 
In several families with a dominant form of retinitis pigmentosa, the mutation L185P in the RDS gene was nonpenetrant in many carriers that were detected by molecular analysis. It was demonstrated later that all the individuals affected with retinitis pigmentosa were also carriers of a mutation in another unlinked gene ROM1. Both carriers of a single mutation, either L185P in periferin/RDS, or a null mutation in ROM1 were healthy. Only the carriers of both mutations (double heterozygotes) were clinically affected with retinitis pigmentosa. ${ }^{12}$ The explanation for these observations is that the encoded proteins of peripherin/RDS and ROM1 form homodimers that interact covalently with each other. ${ }^{13}$ Therefore, mutations in one allele of each of the genes lead to an abnormal complex and eventually to retinitis pigmentosa.

\section{Bardet Biedl syndrome}

Bardet Biedl syndrome (BBS) is an autosomal-recessive disorder including mental retardation, obesity, polydactyly, and retinal degeneration. ${ }^{14}$ At least 7 different loci are known and some of the genes responsible have been characterized. Although segregation analyses in large families were compatible with a classical autosomal recessive inheritance, it seems that the picture may be more complicated. Several of the patients were found to be homozygous at one of the BBS loci and heterozygous at another BBS locus. ${ }^{15}$ This digenic inheritance was referred as triallelic because it is a recessive disorder and the patients carry three mutations. This type of inheritance is responsible for some of the BBS cases, and it has been proposed that it may represent a more general explanation for some syndromes. ${ }^{15}$

\section{Imprinting}

Both parental genomes are essential for normal growth and development. In mice and later in humans, it was demonstrated that some of the genes are imprinted, expressed only from either the maternal or paternal chromosome. A mutation in one of these imprinted genes will be expressed only if present on the chromosome from the parent who transmits the active copy of the gene.

\section{Angelman syndrome}

Angelman syndrome is a disorder characterized by mental retardation, happy disposition, bursts of laugher, puppet-like movements, and wide-based gait. ${ }^{16}$ The syndrome may be caused by a deletion or uniparental disomy of the region 15q11-q13 or by a mutation in the UBE3A gene. Carriers of mutations in the UBE3A gene may or may not present clinical symptoms depending upon the parent from whom they received the mutation. ${ }^{17}$ Only children who received the mutation from their mother present with symptoms of the syndrome. Those children who received the mutation from their father are unaffected, but if they are females, they may transmit the disease to their offspring.

\section{Influence of environmental factors}

For some mutations, the affected individuals present symptoms only after exposure to an environmental factor.

\section{Aminoglycoside-induced mitochondrial deafness}

Familial occurrence of hearing loss after a modest dose of streptomycin along with a mitochondrial inheritance has been observed in several families. ${ }^{18}$ The carriers of the mutation A1555G of the 12sRNA in the mitochondrial DNA are normal and only if there is a second event, such as exposure to aminoglycosides, will the individual be affected. Another probable second event is a mutation in the nuclear DNA. In several families, congenital deafness was observed without evident exposure to drugs, and linkage analysis suggested the existence of a modifier on chromosome $8 .{ }^{19}$

\section{EXPRESSIVITY: DIFFERENCES IN THE SAME PHENOTYPE}

Mutations in the same gene may cause different clinical symptoms and two different groups of expressivity may be distinguished. The same molecular genotype may present differences in either the severity of the same clinical phenotype or differences in the clinical presentation. It is probable that in many cases the combined influence of genetic and environmental factors leads to the difference in expressivity (Table 2).

\section{"Pseudo" differences in expressivity}

Homozygotization of a dominant mutation may give the impression of differences in expressivity because the phenotype of the homozygote is often more severe than the one of the heterozygote. In a family in which an affected individual presents with a severe form of a dominant disease, the possibility of homozygotization may be envisaged if both parents present symptoms. However, this possibility may not be evident either because of incomplete penetrance in one of the parents or uniparental disomy in the child.

Table 2

Examples of mechanisms explaining differences in expressivity

Differences in the same phenotype
"Pseudo" differences in expressivity
Influence of a polymorphism in cis
Influence of the allele in trans
Influence of another gene
Anticipation
Influence of the sex
Somatic mosaicism
Influence of environmental factors
Differences in the clinical presentation
Molecular heterogeneity
Influence of a polymorphism in cis
Imprinting
Somatic mosaicism




\section{Waardenburg syndrome}

Waardenburg syndrome type I is an autosomal-dominant disorder with the classical triad of deafness, pigmentary changes such as a white forelock, and dystopia canthorum. ${ }^{20}$ In a consanguineous couple with a family history of deafness, their first child presented with the classical form of Waardenburg syndrome including a white lock and dystopia canthorum. ${ }^{21}$ The second child was born with a very severe form of the syndrome suggesting variable expressivity. However, clinical examination of the parents revealed that both had dystopia canthorum and were therefore affected. These observations suggested the possibility of homozygotization of the child that was demonstrated by molecular studies. ${ }^{21}$

\section{Influence of a polymorphism present in cis}

Several polymorphisms/mild mutations have been reported that if they are combined in cis they may lead to a severe phenotype.

\section{Cystic fibrosis}

Patients with the genotype DeltaF508/R347H-D979A are affected with a severe form of cystic fibrosis including pancreatic insufficiency and severe pulmonary manifestations. However, the individual mutations R347H and D979A in the CFTR gene are known to be mild and as suggested by the clinical observations, when combined in cis they act as a severe mutation. When these mutants were transiently expressed in HeLa cells, $\mathrm{R} 347 \mathrm{H}$ was associated with defective chloride activity and D979A lead to misprocessing. ${ }^{22}$ The mutant R347H-D979A expressed both defects and dramatically reduced the chloride channel activity.

\section{Influence of the allele in trans}

Either a polymorphism or a mutation in the allele in trans may lead to the difference in the expressivity of a dominant mutation.

\section{Elliptocytosis}

Elliptocytosis is an autosomal-dominant hematological disorder characterized by elliptically shaped erythrocytes and hemolytic anemia. ${ }^{23}$ The disorder results from mutations in one of the genes encoding for the red cell membrane skeleton in particular the $\alpha$ - and the $\beta$-spectrin genes. A relatively common polymorphism lowers the $\alpha$-spectrin gene expression (LE allele). Therefore when the LE allele is present in cis of the mutation for elliptocytosis, the product of the double heterozygote allele will be rejected and only the product of the normal allele will be incorporated. In this case, the polymorphism rescues the individual from the elliptocytosis mutation. However, if the LE allele is present in trans of a mutation for elliptocytosis, it will result in a more severe clinical picture than the one found in patients with the mutation alone. ${ }^{24}$

\section{Influence of another gene}

The clinical presentation of a monogenic disorder may depend upon the presence of a mutation in another gene.

\section{Dominant glaucoma}

Mutations in the two genes MYOC and CYP1B1 have been characterized in some forms of glaucoma: MYOC in autosomal-dominant open angle glaucoma and CYP1B1 in recessive congenital glaucoma. ${ }^{25,26}$ In a single large family, several individuals affected with dominant glaucoma had mutations in both MYOC and CYP1B1. ${ }^{27}$ The clinical presentation was different for patients with a MYOC mutation alone (mean age of onset 51 years) or when the mutation was present together with a CYP1B1 mutation (mean age of onset 27 years). These observations suggested that CYP1B1 acts as a modifier of MYOC and that the two genes interact through a common pathway.

\section{Anticipation}

Anticipation is a term used to describe the transmission of a dominant disorder with an earlier onset and more severe manifestations from one generation to the next.

\section{Myotonic dystrophy}

Myotonic dystrophy is an autosomal-dominant disorder with a wide range of clinical symptoms. The major clinical features include myotonia, cataracts, hypogonadism, frontal balding, and electrocardiographic changes. Some of the patients also present with mental retardation. Anticipation was, at first, a clinical observation but that has been challenged, and it was thought to represent a selection bias. Later studies of families after exclusion of the index patients demonstrated an earlier onset and more severe phenotype among children of almost all of the parent-child pairs. ${ }^{27}$ At the molecular level, the disorders in which clinical anticipation is present are caused by dynamic mutations with anticipation being, at least in part, related to the expansion of the repeat number. ${ }^{28}$

\section{Influence of the sex}

The clinical presentation of a monogenic disorder may depend upon the sex of the affected individual. In some cases, a particular clinical symptom may be present in one sex only.

\section{Cystic fibrosis}

Among affected individuals females are usually fertile and males infertile because of bilateral aplasia of the vas deferens. ${ }^{29}$ Congenital bilateral aplasia of the vas deferens is not a developmental anomaly but a degenerative change of the vas deferens in males due to an obstruction similar to that which occurs in cystic fibrosis in the pancreas and other organs.

\section{Somatic mosaicism}

The presence of a mutation as a somatic and germinal mosaicism often leads to a milder phenotype in the affected individual than in his children. ${ }^{2}$ The affected children present a more severe form of the disorder because the mutation is present in all of their cells. 


\section{Osteogenesis imperfecta type II}

Osteogenesis imperfecta is a heterogeneous group of disorders of the connective tissue characterized mainly by bone fragility and blue sclerae. Type II or osteogenesis imperfecta congenita is a lethal autosomal-dominant disease. Several families have been reported in which individuals with a mild/moderate form of osteogenesis imperfecta had a child with the lethal form. ${ }^{2}$ Molecular analysis demonstrated that the differences in the clinical presentation were due to the presence of the mutation only as a mosaicism in the parent.

\section{Environmental factors}

In many metabolic diseases, the clinical presentation may depend upon the quantity of substrate in the diet.

\section{Hereditary fructose intolerance}

Many of the cases of fructose intolerance are severely ill infants with recurrent hypoglycemia and vomiting. ${ }^{30}$ This usually occurs at the time of weaning when fructose or sucrose is added to the diet and results in marked malnutrition. However, in some cases when fructose is added later and/or in small quantities, the symptoms may be much milder, with a general marked aversion to sweets and fruits.

\section{EXPRESSIVITY: DIFFERENCES IN THE CLINICAL PRESENTATION}

\section{Molecular heterogeneity}

In some cases, the differences in the clinical presentation are secondary to molecular heterogeneity. Some mutations are responsible for a more severe phenotype, whereas other mutations lead to a milder phenotype.

\section{Tay Sachs disease}

Tay Sachs disease is due to the deficiency of the lysosomal enzyme hexosaminidase A. ${ }^{31}$ Complete absence of the enzyme may be secondary to several mutations that in homozygosity or compound heterozygosity leads to the severe infantile form of the disease. Other mutations allow for the synthesis of an enzyme with some residual activity and therefore are responsible for a milder form of the disease usually with adult onset. ${ }^{31}$

\section{Influence of a polymorphism in cis}

A dominant mutation may lead to different phenotypes depending upon the type of polymorphism present in the same gene in cis.

\section{Creutzfeld-Jakob disease and Fatal Familial Insomnia}

Both Creutzfeld-Jakob disease and Fatal Familial Insomnia are rare, autosomal-dominant, phenotypically distinct, and progressive neurological disorders. Although no sleep impairment has been reported in Creutzfeld-Jakob disease, the same mutation at codon 178 of the prion protein gene is responsible for both Creutzfeld-Jakob disease and Fatal Familial Insomnia. A different segregation of the valine-methionine polymor- phism in codon 129 was demonstrated in each of the two diseases (valine in Creutzfeld-Jakob disease and methionine in Fatal Familial Insomnia). ${ }^{32}$ Each different combination of the mutation at codon 178 and the polymorphism in codon 129 produce different conformations of the prion protein with different protease cleavage sites. As a result the abnormal isoforms of the prion protein differ both in the relative abundance of the glycosylated forms and in the size of the protein resistant fragments.

\section{Imprinting}

According to the parent who transmits the mutation, a different clinical presentation may be observed.

\section{Progressive osseous heteroplasia and Albright's hereditary osteodystrophy}

Progressive osseous heteroplasia is a disease including classically congenital malformations of the big toe and progressive heterotopic ossification of the skeletal muscles, tendons, ligaments, and fascia. ${ }^{33}$ Patients affected with Albright's hereditary osteodystrophy have short stature obesity, round face, short fourth metacarpal bones, and often subcutaneous calcifications. ${ }^{34}$ In one family, the same mutation in the GNAS1 gene caused either progressive osseous heteroplasia when maternally inherited or Albright's hereditary osteodystrophy when paternally inherited. ${ }^{35}$ Although several speculations have been proposed, the mechanism is still not understood.

\section{Somatic mosaicism}

The clinical syndrome may be different when the same mutation is present as a somatic mosaicism or in all the body cells.

\section{Epidermal nevus and epidermolytic hyperkeratosis}

Epidermal nevi frequently appear at birth or shortly after. Somatic mosaicism for a mutation in the gene for keratin 10 is responsible for some epidermal nevi. ${ }^{36}$ Mutations in the same gene also cause epidermolytic hyperkeratosis, which is a generalized dermatological disease. Several families have been reported in whom a child was affected with epidermolytic hyperkeratosis due to a keratin 10 mutation in all the cells, whereas an epidermal nevus was found in the parent with the mutation present only in a mosaic state.

\section{CONCLUSION}

Nonpenetrance and differences in expressivity of monogenic disorders in humans have been delineated very early in medical genetics. With the ability to understand the molecular basis for many monogenic diseases, some of the basic processes leading to nonpenetrance and differences in expressivity became clear. Still, for most of the cases, the complete mechanisms explaining either nonpenetrance or differences in expressivity are not understood. Understanding the complexity of the factors leading to nonpenetrance or differences in expressivity in "simple" monogenic traits will serve as a model for revealing the basis of complex traits. 
Although the influence of modifiers and environmental factors on monogenic disorders has been suspected, it is only recently that their molecular basis has begun to be understood. In many other cases, the mechanisms such as imprinting, digenic inheritance, or expanding mutations came as a surprise to the medical geneticists, even though they have been previously described in other organisms.

Almost all the mechanisms to nonpenetrance and expressivity are common; however, the distinction between the two phenomena is still important for genetic counseling. Because Neurofibromatosis type I is a disease with complete penetrance, the demonstration that the parents of an affected child have no clinical symptoms allow giving them a very small recurrence risk. $^{2}$

Understanding the basis for incomplete penetrance in some of the genetic diseases may be the key to prevent their symptoms. For instance, in BRCA2-6174delT Ashkenazi Jewish carriers, the presence of the RAD51-135C allele results in an approximately 4 -fold increase in breast cancer risk. ${ }^{37}$ The biological effect of the polymorphism is not yet known, but it has been suggested that the RAD51 status might be useful in differentiating those carriers most likely to benefit from aggressive prevention measures from those in whom more conservative management would be appropriate. The elucidation of the mechanisms protecting carriers of BRCA mutations from developing cancer may help to develop appropriate preventive drugs. ${ }^{25,30}$

\section{References}

1. Stern C. Principles of human genetics, 3rd ed. San Francisco: WH Freeman and Company, 1973.

2. Zlotogora J. Germline mosaicism. Hum Genet 1998;102:381-386.

3. Tawil R, Figlewicz DA, Griggs RC, Weiffenbach B. Facioscapulohumeral dystrophy: a distinct regional myopathy with a novel molecular pathogenesis. FSH Consortium. Ann Neurol 1998;43:279-282.

4. Sherman SL, Jacobs PA, Morton NE, Froster-Iskenius U, Howard-Peebles PN, Nielsen KB et al. Further segregation analysis of the fragile X syndrome with special reference to transmitting males. Hum Genet 1985;69:289-299.

5. Fu Y-H, Kuhl DP, Pizzuti A, Pieretti M, Sutcliffe JS, Richards S et al. Variation of the CGG repeat at the fragile $\mathrm{X}$ site results in genetic instability: resolution of the Sherman paradox. Cell 1991;67:1047-1058.

6. Thigpen AE, Davis DL, Gautier T, Imperato-McGinley J, Russel DW. Brief report: the molecular basis of steroid $5 \alpha$ reductase deficiency in a large Dominican kindred. N Engl J Med 1994;327:1216-1220.

7. Rivolta C, Sharon D, DeAngelis MM, Dryja TP. Retinitis pigmentosa and allied diseases: numerous diseases, genes and inheritance patterns. Hum Mol Genet 2002; 11:1219-1227.

8. McGee TL, Devoto M, Ott J, Berson EL, Dryja TP. Evidence that the penetrance of mutations at the RP11 locus causing dominant retinitis pigmentosa is influenced by a gene linked to the homologous RP11 allele. Am J Hum Genet 1997;61:1059-1066.

9. Morisette J, Clepet C, Moisan S, Dubois S, Winstall E, Vermeeren D et al. Homozygotes carrying an autosomal dominant TIGR mutation do not manifest glaucoma. Nat Genet 1998;19:319-321.

10. Riazuddin S, Castelein CM, Ahmed ZM, Lalwani AK, Mastroianni MA, Naz S et al. Dominant modifier DFNM1 suppresses recessive deafness DFNB26. Nat Genet 2000;26:431-434.
11. Sohocki MM, Daiger SP, Bowne SJ, Rodriquez JA, Northrup H, Heckenlively JR et al. Prevalence of mutations causing retinitis pigmentosa and other inherited retinopathies. Hum Mutat 2001;17:42-51.

12. Kajiwara K, Berson EL, Dryja TP. Digenic retinitis pigmentosa due to mutations at the unlinked peripherin/RDS and ROM1 loci. Science 1994;264:1604-1608.

13. Loewen CJ, Moritz OL, Molday RS. Molecular characterization of periferin 2 and rom mutants responsible for digenic retinitis pigmentosa. J Biol Chem 2001;276: $22388-22396$.

14. Sheffield VC, Nishimura D, Stone EM. The molecular genetics of Bardet Biedl syndrome. Curr Opin Genet Dev 2001;11:317-321.

15. Katsanis N, Ansley SJ, Badano JL, Eichers ER, Lewis RA, Hoskins BE et al. Triallelic inheritance in Bardet-Biedl syndrome, a Mendelian recessive disorder. Science 2001; 293:2256-2259.

16. Williams CA, Lossie A, Driscoll D. Angelman syndrome: mimicking conditions and phenotypes. Am J Med Genet 2001;101:59-64.

17. Matsuura T, Sutcliffe JS, Fang P, Galjaard R-J, Jiang Y, Benton CS et al. De novo truncating mutations in E6-AP ubiquitin-protein ligase gene (UBE3A) in Angelman syndrome. Nat Genet 1997;15:74-77.

18. Prezant TR, Agapian JV, Bohlman MC, Bu X, Oztas S, Qiu W-Q et al. Mitochondrial ribosomal RNA mutation associated with both antibiotic-induced and non-syndromic deafness. Nat Genet 1993;4:289-294.

19. Bykhovskaya Y, Yang H, Taylor K, Hang T, Tun RYM, Estivill X et al. Modifier locus for mitochondrial DNA disease: linkage and linkage disequilibrium mapping of a nuclear modifier gene for maternally inherited deafness. Genet Med 2001;3:177-180.

20. Read AP, Newton VE. Waardenburg syndrome. J Med Genet 1997;34:656-665

21. Zlotogora J, Lerer I, Bar-David S, Ergaz Z, Abeliovich D. Homozygosity for Waardenburg syndrome. Am J Hum Genet 1995;56:1173-1178.

22. Clain J, Fritsch J, Lehmann-Che J, Bali M, Arous N, Goossens M et al. Two mild cystic fibrosis-associated mutations result in severe cystic fibrosis when combined in cis and reveal a residue important for cystic fibrosis transmembrane conductance regulator processing and function. J Biol Chem 2001;276:9045-9049.

23. Tse WT, Lux SE. Red blood cell membrane disorders. Br J Haematol 1999;10:213.

24. Gratzer. Human genetics: Silence speaks in spectrin. Nature 1994;372:620-621.

25. WuDunn D. Genetic basis of Glaucoma. Curr Opin Ophthalmol 2002;13:55-60.

26. Vincent AL, Billingsley G, Buys Y, Levin AV, Priston M, Trope G et al. Digenic inheritance of early-onset glaucoma: CYP1B1, a potential modifier gene. Am J Hum Genet 2002;70:448-460.

27. Howeler CJ, Busch HF, Geraedts JP, Niermeijer MF, Staal A. Anticipation in myotonic dystrophy: fact or fiction? Brain 1989;112:779-797.

28. Ashizawa T, Dubel JR, Dunne PW, Dunne CJ, Fu YH, Pizzuti A et al. Anticipation in myotonic dystrophy, II: Complex relationships between clinical findings and structure of the GCT repeat. Neurology 1992;42:1877-1883.

29. Oppenheimer EH, Esterly JR. Observations on cystic fibrosis of the pancreas, V: Developmental changes in the male genital system. J Pediat 1996;75:806-811.

30. Perheentupa J, Pikanen E. Symptomless hereditary fructose intolerance. Lancet 1962;1:1358-1359.

31. Myerowitz R. Tay-Sachs disease causing mutations and neutral polymorphisms in the Hex A gene. Hum Mut 1997;9:195-208.

32. Monari L, Chen SG, Brown P, Parchi P, Petersen RB, Mikol J et al. Fatal Familial Insomnia and Familial Creutzfeldt-Jakob Disease: Different prion proteins determined by a DNA polymorphism. Proc Natl Acad Sci U S A 1994;91:2839-2842.

33. Kaplan FS, Shore EM. Progressive osseous heteroplasia. J Bone Miner Res 2000;15: 2084-2094.

34. Wilson LC, Trembath RC. Albright's hereditary osteodystrophy. J Med Genet 1994; 31:779-784.

35. Shore EM, Ahn J, Jan de Beur S, Li M, Xu M, Gardner RJ et al. Paternally inherited inactivating mutations of the GNAS1 gene in progressive osseous heteroplasia. N Engl J Med 2002;346:99-106.

36. Paller AS, Syderr AJ, Chan YM, Hutton E, Tadini G, Fuchs I. Genetic and clinical mosaicism in a type of epidermal nevus. N Engl J Med 1994;331:1447-1449.

37. Levy-Lahad E, Lahad A, Eisenberg S, Dagan E, Paperna T, Kasinetz L et al. A single nucleotide polymorphism in the RAD51 gene modifies cancer risk in BRCA2 but not BRCA1 carriers. Proc Natl Acad Sci U S A 2001;98:3232-3236. 